\title{
Single frequency Tm-doped fibre DBR laser at 1943 nm
}

\author{
Z. Zhang, A. J. Boyland, J. K. Sahu, W. A. Clarkson and M. Ibsen \\ Optoelectronics Research centre, University of Southampton, Southampton, SO17 1BJ UK
}

Stable single frequency two-micron lasers are attractive sources for numerous application areas, including spectroscopy, gas sensing and nonlinear frequency conversion [1-2]. In this paper we report on a Tm-doped silica fibre laser, based on a short cavity distributed Bragg reflector (DBR) design, employed in a simple masteroscillator power-amplifier configuration to produce $2.8 \mathrm{~W}$ of stable, linearly-polarised single-frequency output at $1943 \mathrm{~nm}$.

The Tm-doped fibre DBR laser was constructed from photosensitive polarization-maintaining (PM) and nonPM Tm fibre. Both fibres were fabricated in-house starting with the same perform to give nearly identical core composition in the two fibres. Each fibre had a Tm-Ge co-doped alumino-silicate core with a core diameter of $\sim 10 \mu \mathrm{m}$ and a numerical aperture (NA) of $\sim 0.17$. A relatively high Tm concentration ( 1 wt. $\%$ ) was used to allow efficient absorption of pump light at $1565 \mathrm{~nm}$ in a short length of fibre. Fig. 1(a) shows the Tm fibre DBR laser structure. A $3 \mathrm{~cm}$ long high reflector (HR) fibre Bragg grating (FBG) was written in the non-PM Tm fibre, with a $3 \mathrm{~dB}$ bandwidth of $\sim 6 \mathrm{GHz}$ (see Fig. 1(b)), and a $2 \mathrm{~cm}$ long output coupler (OC) FBG was written in the PM Tm fibre. The transmission spectra for the two gratings are shown in Fig. 1(b). The OC grating has two transmission dips corresponding to orthogonal polarisations and the centre wavelengths of the HR and OC gratings were slightly different. The HR and OC gratings were fusion spliced together with a $\sim 1 \mathrm{~cm}$ long length of Tm fibre between the gratings. The effective length of the DBR cavity was $\sim 3 \mathrm{~cm}$, corresponding to a longitudinal-mode-spacing of $\sim 3.3 \mathrm{GHz}$. Hence, the DBR cavity could support no more than two longitudinal modes. A linearly-polarised single-longitudinal-mode output could be selected by simply adjusting the temperature of either grating.

The DBR laser was pumped by an Er/Yb co-doped single-transverse-mode fibre laser with a central wavelength at $\sim 1565 \mathrm{~nm}$. At the pump input end, a dichroic mirror was used to separate the backward output signal from the DBR laser. Around half of the pump power was absorbed by the DBR structure. Therefore, a $60 \mathrm{~cm}$ long PM Tm fibre was spliced after the OC FBG to absorb the remaining (unabsorbed) pump power from the DBR to act as simple amplifier for the forward output signal from the DBR.
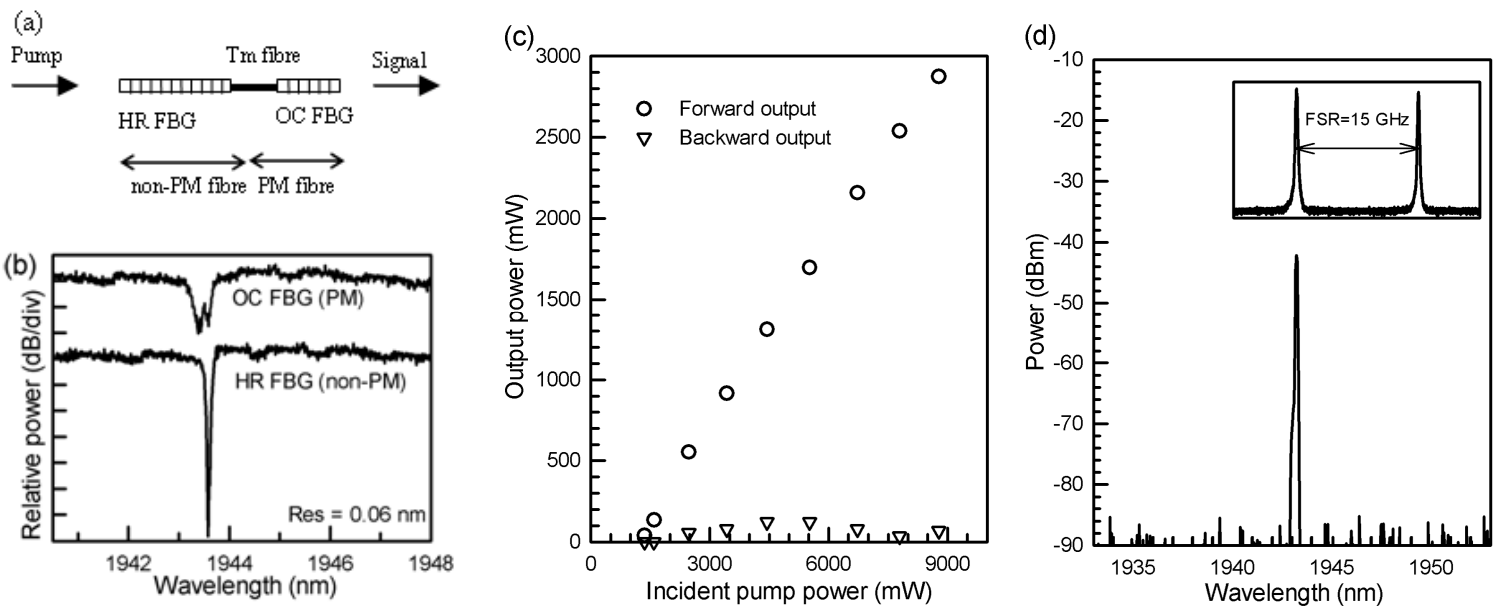

Fig. 1 (a) Tm DBR fibre laser structure, (b) measured transmission spectra of the HR and OC FBGs, (c) output power from the DBR MOPA, and (d) emission spectrum (inset: a scanning Fabry-Perot interferometer trace).

The output power from the DBR MOPA is shown in Fig.1(c). Threshold for DBR lasing was reached at an absorbed pump power of $\sim 800 \mathrm{~mW}$. At an input pump power of $9 \mathrm{~W}$, the forward output power from the MOPA was $\sim 2.8 \mathrm{~W}$. The MOPA slope efficiency for the forward output power with respect to incident pump power was $\sim 38 \%$. The nonlinear behavior of the backward output power with respect to incident pump power is believed to be due to thermal effects in the DBR laser. Fig.1(d) shows the emission spectrum centred at $\sim 1943 \mathrm{~nm}$. The measured side mode suppression ratio of $40 \mathrm{~dB}$ was limited by the sensitivity of the optical spectum analyzer. Single frequency operation of the DBR laser was confirmed with the aid of a scanning Fabry-Perot interferometer with a FSR of $15 \mathrm{GHz}$ and finesse of 100 (inset of Fig.1(d)), and the polarisation extinction ratio was measured to be $>20 \mathrm{~dB}$. The prospects for further improvement in performance will be discussed.

\section{References}

[1] S. Agger, J. H. Povlsen, and P. Varming, Optics Letters 29, 150 (2004).

[2] J. Geng, J. Wu, S. Jiang and J. Yu, Optics Letters 32, 355 (2007). 